\title{
Effects of Nonuniform Outflow and Buoyancy on Drag Coefficient Acting on a Spherical Particle
}

\author{
Mariko Watanabe, Joji Yahagi \\ Faculty of Science and Technology, Sophia University, Tokyo, Japan \\ Email: mariko_w@sophia.ac.jp
}

How to cite this paper: Watanabe, M. and Yahagi, J. (2017) Effects of Nonuniform Outflow and Buoyancy on Drag Coefficient Acting on a Spherical Particle. Journal of Flow Control, Measurement \& Visualization, 5, 99-110.

https://doi.org/10.4236/jfcmv.2017.54008

Received: May 29, 2017

Accepted: October 16, 2017

Published: October 19, 2017

Copyright $\odot 2017$ by authors and Scientific Research Publishing Inc. This work is licensed under the Creative Commons Attribution International License (CC BY 4.0).

http://creativecommons.org/licenses/by/4.0/

\begin{abstract}
Pyrolysis gas jets out from the surface of a solid fuel particle when heated. This study experimentally observes the occurrence of gas jets from heated solid fuel particles. Results reveal a local gas jet occurs from the particle's surface when its temperature reaches the point at which a pyrolysis reaction occurs. To investigate the influence of the gas jet on particle motion, a numerical simulation of the uniform flow around a spherical particle with a nonuniform outflow or high surface temperature is conducted, and the drag force acting on the spherical particle is estimated. In the numerical study, the magnitude of the outflow velocity, direction of outflow, and Rayleigh number, i.e., particle surface temperature, are altered, and outflow velocities and the Rayleigh number are set based on the experiment. The drag coefficient is found to decrease when an outflow occurs in the direction against the mainstream; this drag coefficient at a higher Rayleigh number is slightly higher than that at a Rayleigh number of zero.
\end{abstract}

\section{Keywords}

Drag Force, Particle, Nonuniform Outflow, Heat

\section{Introduction}

Pulverized coal is mixed with air and burned in pulverized coal combustion devices in power plants and ironworks. When a solid fuel particle such as coal is heated, the flammable gas generated by the pyrolysis reaction jets out from a part of the surface, and combustible components that are not gasified burn at the surface. Although the reaction rate and yields of each component in such a combustion process have been frequently investigated [1]-[6], few studies have 
examined the fluid-dynamic behavior of the gas jet and the effects of the gas jet on the surrounding flow and solid fuel particle motion.

In a numerical simulation of a gas-particle flow, an equation has been solved for the motion of a particle in which drag, gravitational, and other forces are included [7] [8]. An equation of the drag coefficient is important to accurately estimate particle trajectories, and many studies have been conducted on drag forces acting on a particle in a flow field. For example, Haider and Levenspiel proposed equations for the drag coefficient of spherical and nonspherical particles [9], and numerical simulations have been conducted to investigate the drag forces acting on a nonspherical particle [10] [11]. Lee and Balachandar estimated drag forces on a translating and rotating particle in a wall-bounded linear shear flow [12], and drag forces on a particle with a uniform outflow from the surface were studied by Kurose et al. [13]. Furthermore, Katoshevski et al. experimentally investigated the relation between particle temperature and drag forces; the results showed that the drag force acting on a heated particle increased due to free convection around the particle [14].

The aims of the present work are to clarify the flow around a burning solid fuel particle and to estimate the drag coefficient of the particle. In this paper, a gas jet from a heated coal particle is observed and the gas velocity of the jet and surface temperature of the particle are measured. In addition, the effect of the gas jet and surface temperature on the drag coefficient of a particle are numerically investigated; the numerical simulation supposes a particle Reynolds number for micron-sized particles in pulverized coal combustion and is therefore conducted at low Reynolds numbers $(\mathrm{Re}=10$ and 200).

\section{Experimental Observation of Coal Particle Combustion}

\subsection{Experimental Apparatus}

Figure 1 shows (a) the schematic of the experimental apparatus and (b) a photograph of a coal particle set on a pedestal. The coal particle was placed on a circular pedestal with a diameter of $40 \mathrm{~mm}$ and heated using a spot-type halogen lamp heater (Fintech Co., Ltd., HSH-30/f30/24V-75W, spot diameter of $7 \mathrm{~mm}$, power density of $40 \mathrm{~W} / \mathrm{cm}^{2}$ ). The distance between the coal particle and the halogen lamp heater was $30 \mathrm{~mm}$. Table 1 shows the characteristics of the coal. A high-speed camera (DITECT Co., Ltd., HAS-D71), macro zoom lens (Kowa Optical Products Co., Ltd., LMZ45T3), YVO4 laser (KATOKOKEN Co., Ltd., PIV Laser G1000, wavelength of $532 \mathrm{~nm}$ ), and an interference filter (SIGMAKOKI Co., Ltd., center wave-length of $530 \mathrm{~nm}$, half-value width of 10 $\mathrm{nm}$, lower limit of transmittance of 55\%) were used to observe gas jets from the coal particle and measure the gas jet velocity using a spatial resolution of $480 \times$ 640 pixels for an area of approximately $30 \times 40 \mathrm{~mm}$ and a frame rate of $4000 \mathrm{fps}$. Gas jet velocity vectors were measured by particle image velocimetry (PIV) using commercial software (DITECT Co., LTD., Flownizer 2D). Prior to measuring the gas jet velocity, the uniform flow velocity was measured by PIV, and the 


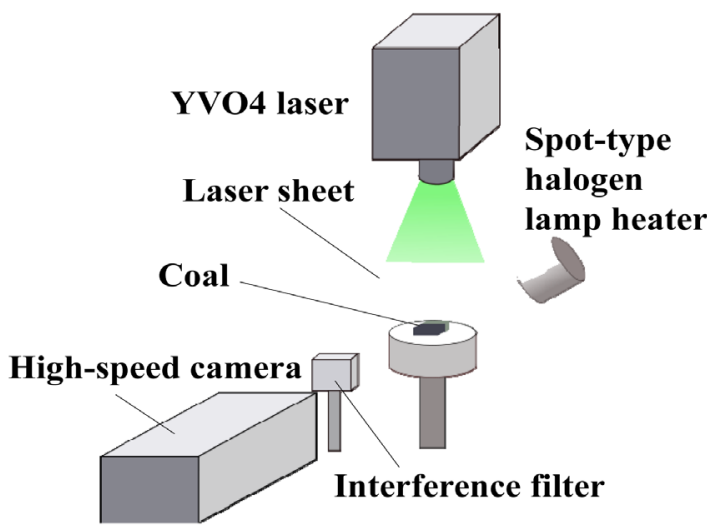

(a)

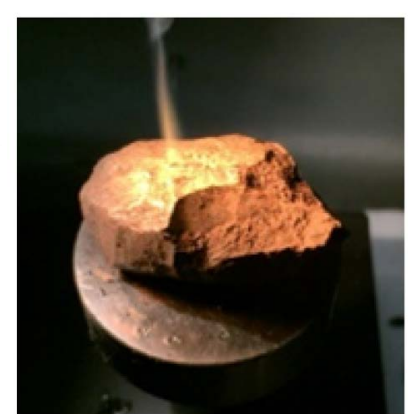

(b)

Figure 1. (a) Experimental setup and (b) a coal particle.

Table 1. Characteristics of coal.

\begin{tabular}{cc}
\hline Type & Senakin coal (bituminous coal) \\
\hline Water & $3.9 \%$ \\
Ash & $12.2 \%$ \\
Volatile Component & $43.3 \%$ \\
Fixed Carbon & $40.5 \%$ \\
\hline
\end{tabular}

velocity obtained was compared to that measured with a Pitot tube; the difference between both values was $7 \%$ or less and thus velocity measurements were deemed to be valid. At the point at which gas jetted out from the coal, the temperature of the coal particle was measured using infrared thermography (Nippon Avionics Co., Ltd., InfRec Thermo GEAR G120EX) with a spatial resolution of $340 \times 240$ pixels for an area approximately $100 \times 70 \mathrm{~mm}$, and a large measuring accuracy of $\pm 2^{\circ} \mathrm{C}$ and $2 \%$ was used for the measured temperature.

\subsection{Experimental Results}

Figure 2 shows a photograph of a jet occurring from the coal particle. In the initial stages of solid fuel combustion, volatile components generated at a relatively low temperature gasify and burn in a state of gas. The material with a low melting point melts and closes off the path of gas during this process; therefore, the internal pressure increases until gas breaks through the liquid film and jets out [15]. The surface part of the particle is shown in white in Figure 2 (the particle received light from the laser sheet). A mushroom-like smoke was seen to jet out from the particle surface, as shown in the red circle. Although Figure 2 shows only one jet, multiple jets actually occurred at different positions on one sample. PIV was conducted using white smoke as a tracer, which was released when the coal particle was heated. The white smoke comprises water droplets generated by the oxidation reaction between oxygen in the air and hydrogen from the pyrolysis reaction. Table 2 shows the size, jet velocity, and surface temperature of three samples, where size refers to the side length of the cuboid circumscribed in the 


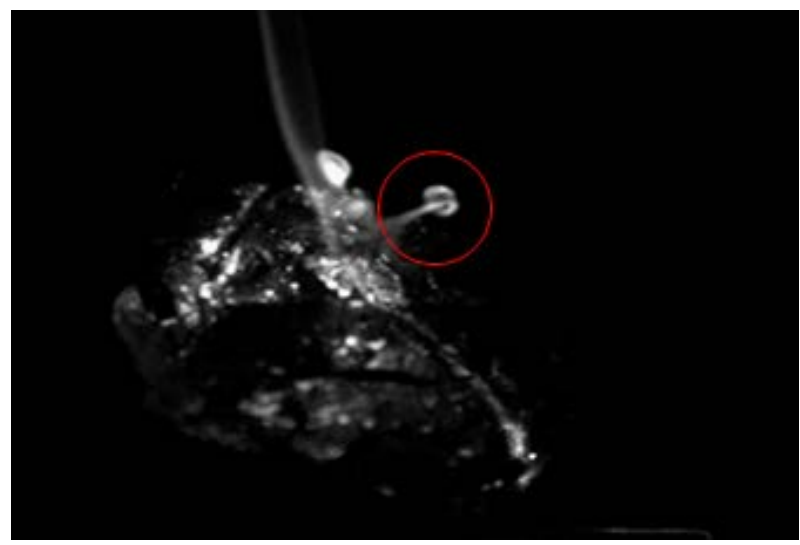

Figure 2. Jet from coal particle.

Table 2. Size, jet velocity, and surface temperature of three coal samples.

\begin{tabular}{cccc}
\hline & Size $[\mathrm{mm}]$ & Jet Velocity $[\mathrm{m} / \mathrm{s}]$ & Surface Temperature $[\mathrm{K}]$ \\
\hline Sample 1 & $36 \times 40 \times 15$ & 1.50 & 632.0 \\
Sample 2 & $27 \times 22 \times 20$ & 1.87 & 717.3 \\
Sample 3 & $23 \times 15 \times 5$ & 1.70 & 671.8 \\
\hline
\end{tabular}

sample, the jet velocity is the arithmetical mean value of maximum and minimum velocity vectors of the jet, and surface temperature is that of a cell located at the center of the jet taken by an infrared thermography image when the gas had begun to release. Three samples of different sizes were found to have similar jet velocities and surface temperatures of approximately $674 \mathrm{~K}$; this temperature is within the temperature range at which coal pyrolysis reactions take place.

\section{Calculation Method}

The gas jet was experimentally confirmed to have occurred locally due to a pyrolysis reaction when the coal particle was heated and the surface temperature of the particle increased, as mentioned above. However, using this experiment it was difficult to determine the influence of the jet occurring from the particle surface on the drag forces acting on the particle. The effects of nonuniform outflows and surface temperatures on drag forces acting on the particle were therefore investigated using a numerical simulation as follows.

\subsection{Governing Equations}

In this study, OpenFOAM-2.3.1 was used for computational fluid dynamics. The continuity equation, the Navier-Stokes equation, and the energy conservation equation are expressed as follows,

$$
\begin{gathered}
\frac{\partial \rho}{\partial t}+\nabla \cdot(\rho \mathbf{u})=0 \\
\frac{\partial(\rho \boldsymbol{u})}{\partial t}+\nabla \cdot(\rho \boldsymbol{u u})=-\nabla p+\nabla \cdot\left[\mu\left\{\nabla \boldsymbol{u}+(\nabla \mathbf{u})^{\mathrm{T}}\right\}\right]-\nabla\left(\frac{2}{3} \mu \nabla \cdot u\right)+\rho \beta\left(T-T_{0}\right) \boldsymbol{g}
\end{gathered}
$$




$$
\frac{\partial(\rho h)}{\partial t}+\nabla \cdot(\rho h \boldsymbol{u})+\frac{\partial(\rho K)}{\partial t}+\nabla \cdot(\rho K \boldsymbol{u})=\frac{\partial p}{\partial t}+\nabla \cdot(k \nabla T),
$$

where $\rho$ is density at a standard temperature, $T_{0} ; \boldsymbol{u}$ is the velocity vector; $p$ is pressure; $\mu$ is viscosity; $\beta$ is the thermal expansion coefficient; $T$ is temperature; $\boldsymbol{g}$ is the gravitational acceleration vector; $h$ is enthalpy; $K$ is kinetic energy; and $k$ is thermal conductivity. An incompressible flow was assumed, and $\rho$ in the above equations was constant.

\subsection{Pressure and Friction Drag Coefficients}

The pressure drag coefficient $C_{D \mathrm{p}}$ is expressed as follows,

$$
C_{D \mathrm{p}}=\frac{2 D_{\mathrm{p}}}{\rho|\boldsymbol{U}|^{2} S}=\frac{2 \int_{A}-p n_{x} \mathrm{~d} A}{\rho|\boldsymbol{U}|^{2} S},
$$

where $D_{\mathrm{p}}$ is the pressure drag; $|U|$ is the magnitude of mainstream; $S$ is the projected area of sphere; $n_{x}$ is the $\mathrm{x}$-direction component of unit normal vector at each point on the sphere; and $A$ is the surface area of sphere. In this calculation, $D_{\mathrm{p}}$ was calculated from the following equation,

$$
D_{\mathrm{p}}=\sum_{i}-p_{i} \frac{r_{i x}}{r} A_{i}
$$

where $p_{i}$ is pressure of the $i$-th grid on the sphere surface; $r_{i x}$ is the x-direction component of the position vector of the $i$-th grid from the center of the sphere; $r$ is the radius of the sphere; and $A_{i}$ is the area of the $i$-th grid. The friction drag coefficient, $C_{D \text { f }}$, is expressed as follows,

$$
C_{D \mathrm{f}}=\frac{2 D_{\mathrm{f}}}{\rho|\boldsymbol{U}|^{2} S}=\frac{2 \int_{A} \tau_{x} \mathrm{~d} A}{\rho|\boldsymbol{U}|^{2} S},
$$

where $D_{\mathrm{f}}$ is the friction drag and $\tau_{x}$ is the x-direction component of shear stress acting on the sphere surface. This calculation assumes a surface of a virtual sphere with a radius of $1.01 r$. The tangential component of velocity at each grid on the virtual sphere surface, $u_{\mathrm{t} p}$, was examined. Since flow velocity was zero on the (actual) sphere surface, and the distance between the virtual and the (actual) sphere surfaces was $0.01 r$, the friction drag was calculated from the following equation,

$$
D_{\mathrm{f}}=\sum_{i} \mu \frac{u_{\mathrm{ti}}}{0.01 r} A_{i}
$$

\subsection{Calculation Domain}

The three-dimensional flow around a rigid sphere of diameter, $d$, fixed in a uniform mainstream, $U$, was calculated. The calculation domain is shown in Figure 3, and is a rectangular parallelepiped of $40 d \times 20 d \times 20 d$. The sphere was placed at the center of the calculation domain, and the origin of the coordinate system was set as the center of the sphere. 


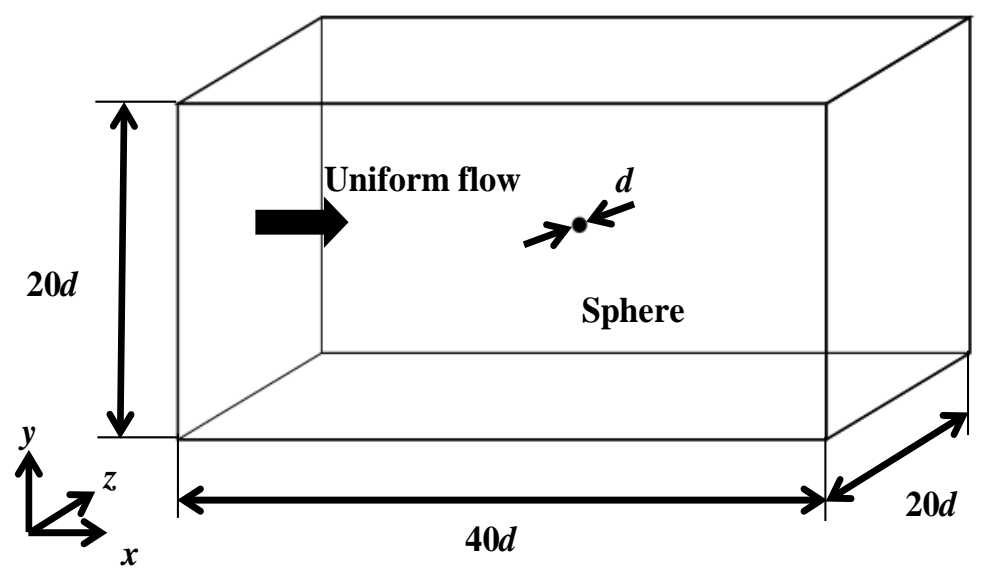

Figure 3. Calculation domain.

\subsection{Calculation Conditions}

The governing equations were discretized through a finite volume method, and the merged PISO-SIMPLE (called PIMPLE in OpenFOAM) algorithm was used for pressure-velocity coupling. The calculation domain was divided into approximately 320,000, nonuniformly-spaced computational cells, as shown in Figure 4. A second-order central difference scheme was applied for spatial derivative terms, and time discretization was based on an implicit Euler scheme. The uniform mainstream, $U$, was supplied from the inlet boundary. A Neumann condition of a zero-velocity gradient was employed as the outlet boundary condition. For calculations on the particle with an outflow from the surface, the Reynolds number, Re, which is based on the magnitude of the mainstream and the particle diameter, was set to 10 . To investigate the effects of a nonuniform outflow from the particle surface, six different outflow directions were assumed. Figure 5 shows the locations of the six outflow directions and the outflow velocity distribution of $\mathrm{x}+$ case. The maximum outflow velocities normalized by $|\mathrm{U}|$ were $0.4,0.7,1.0,1.3$, and 1.6; these normalized calculated conditions correspond to the case of a particle at a submicron order, with the outflow velocity obtained from the experiment mentioned above. Calculations were performed using $\mathrm{Re}=200$ for the particle with a high temperature. The Rayleigh number, Ra, is defined as

$$
\mathrm{Ra}=\frac{g \beta \Delta T L^{3}}{v \alpha},
$$

where $g$ is gravitational acceleration; $\Delta T$ is the temperature difference between particle surface and fluid; $L$ is the representative length; $v$ is the kinematic viscosity; and $\alpha$ is thermal diffusivity. When the physical properties of air at normal temperature were used for $\beta$, $v$, and $\alpha$, and the measurement values were used for $\Delta T$ and $L$, an experimental Ra number with an order of $10^{5}$ was obtained. The Rayleigh number in the calculation was set to $10^{5}$, which had the same order as that of the experimental Ra number. The end time of calculations, $t_{\text {end }}$, was 


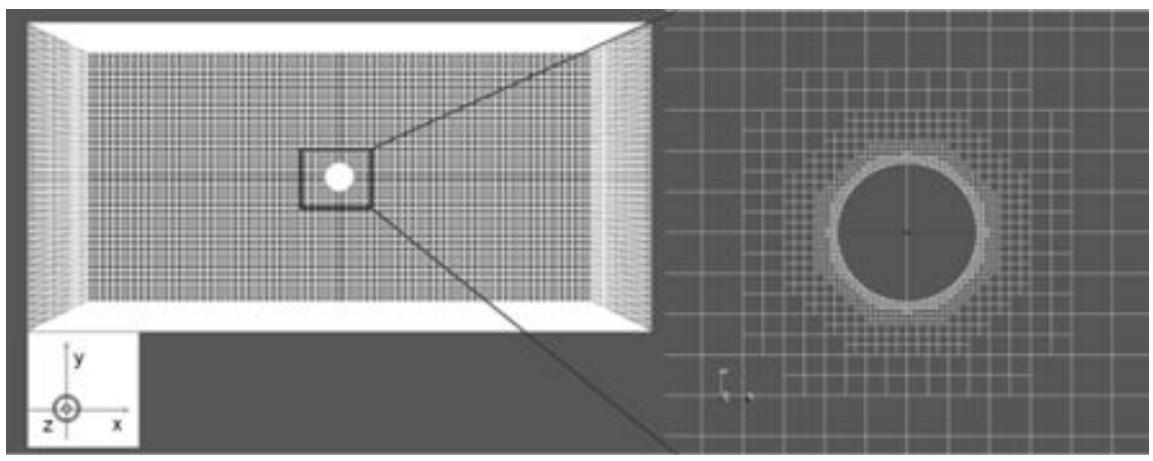

Figure 4. Calculation grids.

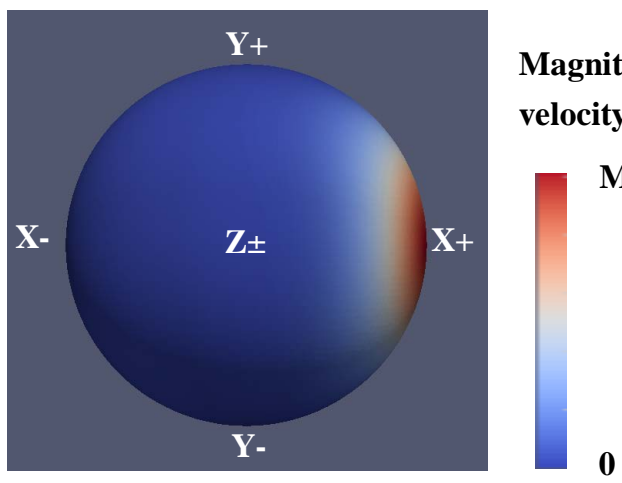

Magnitude of outflow elocity [a.u.]

Maximum velocity

Figure 5. Locations of six outflow directions and outflow velocity distribution of $x+$ case.

decided so that $t_{\text {end }}$ satisfied $|\mathrm{U}| t_{\text {end }} / 40 d>2$. The flow surrounding a particle with no outflow at $\mathrm{Re}=0.4$ was calculated in advance. As the drag coefficient was in agreement with one of Stokes' laws, the calculation method was confirmed to be valid.

\section{Calculation Results and Discussion}

\subsection{Effect of Nonuniform Outflow from Particle Surface}

The results are shown only at $t_{\text {end }}$, as the flow came up at a steady state. Figure 6 and Figure 7 illustrate changes in the pressure and friction drag coefficients, $C_{D \mathrm{p}}$ and $C_{D f}$, with an increase in the normalized maximum outflow velocity, $V$. Figure 8 shows the pressure distribution along the particle surface at $z=0$ at angles ranging from zero to $\pi$; an angle of zero is consistent with the positive $\mathrm{x}$-axis direction, which is counterclockwise. Pressure is normalized by the stagnation pressure in a case of $V=0$. Pressure fluctuation was found in Figure 8 because the boundary of the sphere surface was approximated using a series of rectangular steps without using body-fitted grids. $C_{D \mathrm{p}}$ decreased in a negative x-direction outflow. As the outflow velocity increased, upstream pressure decreased, as shown in Figure 8; thus, pressure drag also decreased. In the positive $\mathrm{x}$-direction outflow, a pressure of around zero rad increased at $V=1$ but decreased at $V=$ 1.6, as shown in Figure 8. Therefore, $C_{D \mathrm{p}}$ decreased until $V=1$ and then increased. $C_{D p}$ gradually increased with outflow toward the y and z directions. $C_{D f}$ 


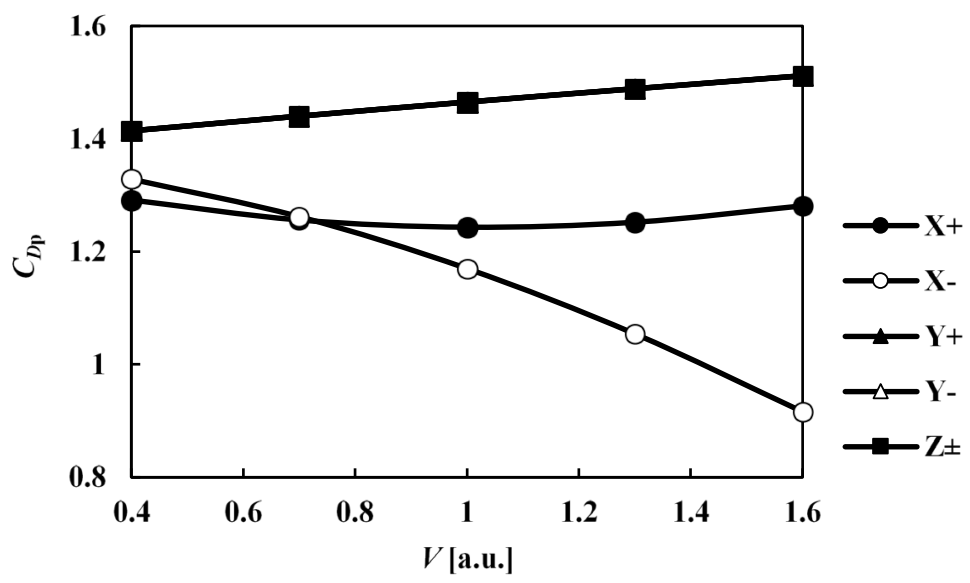

Figure 6. Effects of outflow direction and velocity on pressure drag coefficient.

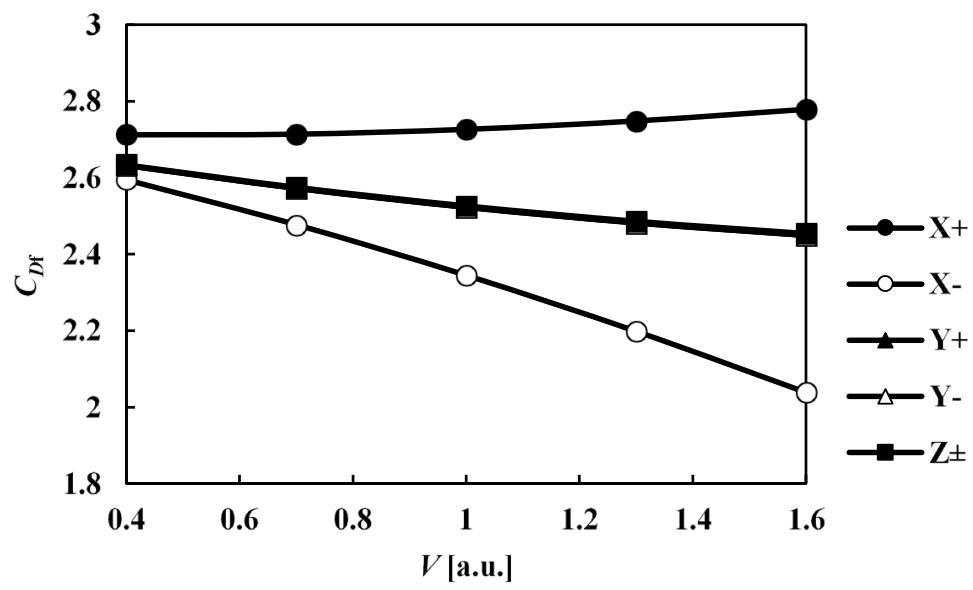

Figure 7. Effects of outflow direction and velocity on friction drag coefficient.

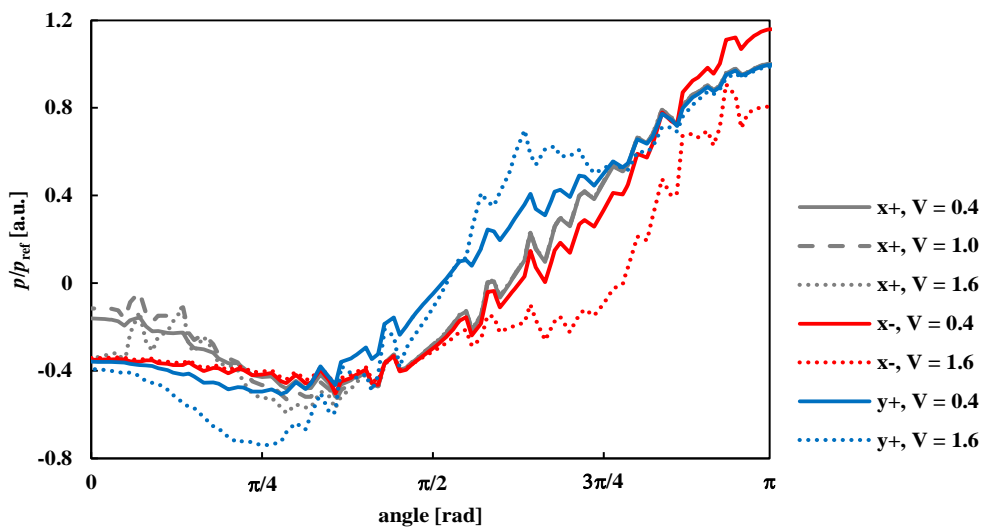

Figure 8. Pressure distribution along particle surface at $\mathrm{z}=0$ and at an angle range from zero to $\pi$.

also largely decreased with a negative $\mathrm{x}$-direction outflow, increased with a positive $\mathrm{x}$-direction outflow, but decreased in the event of $\mathrm{y}$ - and $\mathrm{z}$-direction outflow. Figures $9(a)-(c)$ show the velocity magnitude and streamline when the particle 


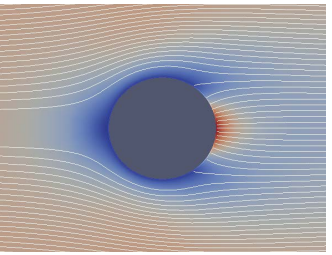

(a)

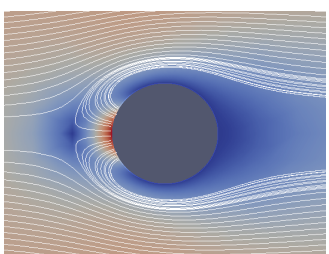

(b)

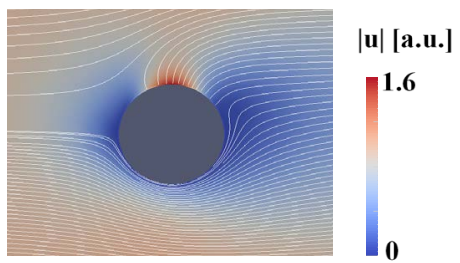

(c)

Figure 9. Velocity magnitude and streamline when particle has (a) positive x-direction outflow, (b) negative $\mathrm{x}$-direction outflow, and (c) positive y-direction outflow.

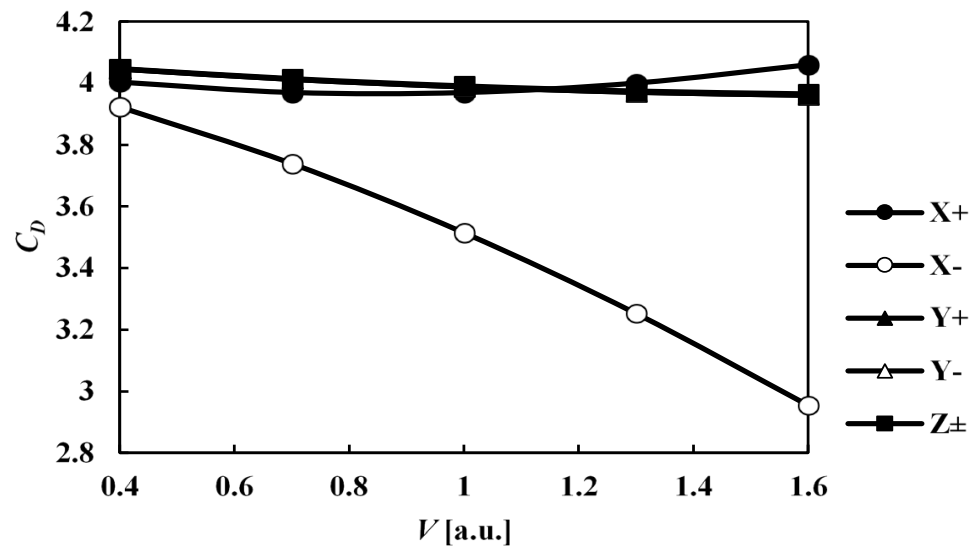

Figure 10. Effects of outflow direction and velocity on drag coefficient.

had a positive $\mathrm{x}$-direction outflow, negative $\mathrm{x}$-direction outflow, and positive $y$-direction outflow, respectively. A separated flow was restrained by the positive $\mathrm{x}$-direction outflow, as shown in Figure 9(a) and the friction drag forces increased. The negative $\mathrm{x}$-direction outflow reduced the pressure drag forces because the stagnation point moved toward the direction due to collisions between the outflow and the mainstream, as shown in Figure $9(\mathrm{~b})$, and the pressure then decreased at the upper side of the particle. It can also be seen in Figure 9(b) that separation of the mainstream occurred due to the negative $\mathrm{x}$-direction outflow. As the separated flow was generated by $y$ - and z-direction outflow, as shown Figure $9(\mathrm{c})$, pressure drag increased and friction drag decreased. Figure 10 shows the relationship between the drag coefficient, $C_{D}$ and $V$. $C_{D}$ decreased in the event of a negative $\mathrm{x}$-direction outflow and slightly increased in the event of a positive x-direction outflow. For the $\mathrm{y}$ - and z-direction outflow events, $C_{D}$ hardly changed because changes in $C_{D \mathrm{p}}$ and $C_{D \mathrm{f}}$ cancelled each other out.

\subsection{Effect of Particle Surface Temperature}

Table 3 shows $C_{D \mathrm{p}}, C_{D \mathfrak{p}}$ and $C_{D}$ at $\mathrm{Ra}=0$ and $10^{5}$. Both $C_{D \mathrm{p}}$ and $C_{D \mathrm{f}}$ increased with increases in Ra. Figure 11 and Figure 12 show the streamline and pressure distribution around the particle at $\mathrm{Ra}=0$ and $10^{5}$. The fluid flowed closely along the particle surface in the lower position at $\mathrm{Ra}=10^{5}$, which caused an increase in the friction drag forces. The lower pressure region moved upwards and expanded at $\mathrm{Ra}=10^{5}$ in comparison with $\mathrm{Ra}=0$; hence, $C_{D p}$ increases when the Ra value is high. 


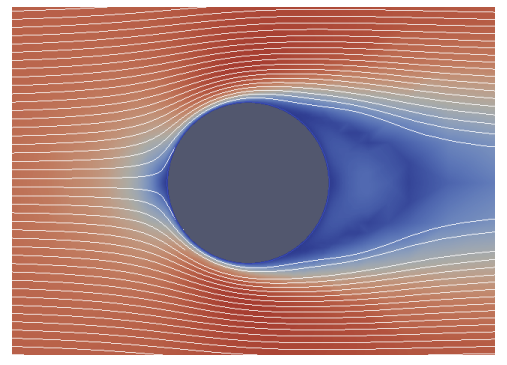

(a)

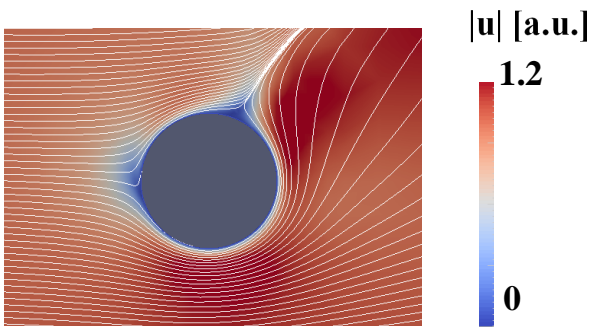

(b)

Figure 11. Streamline and velocity magnitude around particle at (a) $\mathrm{Ra}=0$; (b) $\mathrm{Ra}=10^{5}$.

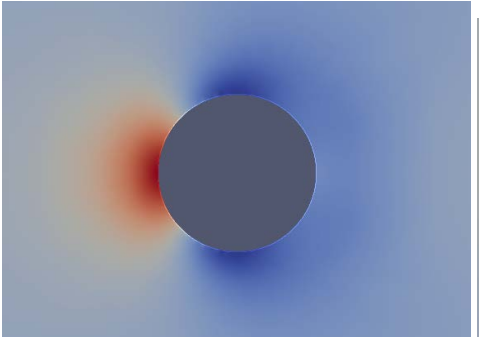

(a)

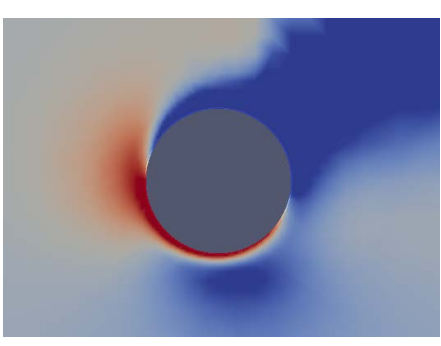

$p / p_{\text {ref }}[$ a.u.]

1.0

(b)

Figure 12. Pressure distribution around particle at (a) $\mathrm{Ra}=0$; (b) $\mathrm{Ra}=10^{5}$.

Table 3. Drag coefficients acting on spherical particle at $\operatorname{Re}=200$.

\begin{tabular}{cccc}
\hline $\mathrm{Ra}$ & $C_{D \mathrm{p}}$ & $C_{D \mathrm{f}}$ & $C_{D}$ \\
\hline 0 & 0.47 & 0.38 & 0.85 \\
$10^{5}$ & 1.12 & 0.59 & 1.71 \\
\hline
\end{tabular}

\section{Conclusion}

This study experimentally observed gas jets from a coal particle surface when the particle was heated and numerically investigated the effects of nonuniform outflows and particle temperatures on drag coefficients acting on a spherical particle. The experimental results show that a jet-like outflow occurred from the particle's surface when the particle was heated to approximately $674 \mathrm{~K}$, and the velocity magnitude of the outflow was approximately $1.7 \mathrm{~m} / \mathrm{s}$. From numerical results, the pressure and friction drag coefficients were found to clearly decrease when an outflow occurred in a direction against the mainstream. It was also found that when the Rayleigh number was high, i.e., the particle surface temperature was high, the drag coefficient was slightly higher than one at $\mathrm{Ra}=$ 0 .

\section{Acknowledgements}

This work was supported by KAKENHI (25630055). We would like to thank Mr. Daiki Ajima for his help with data processing, and Mr. Yuma Onuki and Mr. Shota Iwai for their help while conducting experiments. 


\section{References}

[1] Gomez, C.O. and Vastola, F.J. (1985) Ignition and Combustion of Single Coal and Char Particles. Fuel, 64, 558-563. https://doi.org/10.1016/0016-2361(85)90093-6

[2] Solomon, P.R., Serio, M.A. and Suuberg, E.M. (1992) Coal Pyrolysis: Experiments, Kinetic rates and Mechanisms. Progress in Energy and Combustion Science, 18, 133-220. https://doi.org/10.1016/0360-1285(92)90021-R

[3] Seebauer, V., Petek, J. and Staudinger, G. (1997) Effects of Particle Size, Heating Rate and Pressure on Measurement of Pyrolysis Kinetics by Thermogravimetric Analysis. Fuel, 76, 1277-1282. https://doi.org/10.1016/S0016-2361(97)00106-3

[4] Kök, M.V., Özbas, E., Karacan, O. and Hicyilmaz, C. (1998) Effect of Particle Size on Coal Pyrolysis. Journal of Analytical and Applied Pyrolysis, 45, 103-110. https://doi.org/10.1016/S0165-2370(98)00063-1

[5] Žajdlík, R., Jelemenský, L’, Remiarová, B. and Markoš, J. (2001) Experimental and Modelling Investigations of Single Coal Particle Combustion. Chemical Engineering Sciences, 56, 1355-1361. https://doi.org/10.1016/S0009-2509(00)00358-4

[6] Bejarano, P.A. and Levendis, Y.A. (2008) Single-Coal-Particle Combustion in $\mathrm{O}_{2} / \mathrm{N}_{2}$ and $\mathrm{O}_{2} / \mathrm{CO}_{2}$ Environments. Combustion and Flame, 153, 270-287.

https://doi.org/10.1016/j.combustflame.2007.10.022

[7] Franchetti, B.M., Cavallo Marincola, F., Navarro-Martinez, S. and Kempf, A.M. (2013) Large Eddy Simulation of a Pulverized Coal Jet Flame. Proceedings of the Combustion Institute, 34, 2419-2426. https://doi.org/10.1016/j.proci.2012.07.056

[8] Warzecha, P. and Boguslawski, A. (2014) LES and RANS Modeling of Pulverized Coal Combustion in Swirl Burner for Air and Oxy-Combustion Technologies. Energy, 66, 732-743. https://doi.org/10.1016/j.energy.2013.12.015

[9] Haider, A. and Levenspiel, O. (1989) Drag Coefficient and Terminal Velocity of Spherical and Nonspherical Particles. Powder Technology, 58, 63-70. https://doi.org/10.1016/0032-5910(89)80008-7

[10] Hölzer, A. and Sommerfeld, M. (2009) Lattice Boltzmann Simulations to Determine Drag, Lift and Torque Acting on Non-Spherical Particles. Computers \& Fluids, 38, 572-589. https://doi.org/10.1016/j.compfluid.2008.06.001

[11] Zastawny, M., Mallouppas, G., Zhao, F. and Wachem, B. (2012) Derivation of Drag and Lift Force and Torque Coefficients for Non-spherical Particles in Flows. International Journal of Multiphase Flow, 39, 227-239. https://doi.org/10.1016/j.ijmultiphaseflow.2011.09.004

[12] Lee, H. and Balachandar, S. (2010) Drag and Lift Forces on a Spherical Particle Moving on a Wall in a Shear Flow at Finite Re. Journal of Fluid Mechanics, 657, 89-125. https://doi.org/10.1017/S0022112010001382

[13] Kurose, R., Makino, H., Komori, S., Nakamura, M., Akamatsu, F. and Katsuki, M. (2003) Effects of Outflow from the Surface of a Sphere on Drag, Shear Lift, and Scalar Diffusion. Physics of Fluids, 15, 2338-2351. https://doi.org/10.1063/1.1591770

[14] Katoshevski, D., Zhao, B., Ziskind, G. and Bar-Ziv, E. (2001) Experimental Study of the Drag Force Acting on a Heated Particle. Journal of Aerosol Science, 32, 73-86. https://doi.org/10.1016/S0021-8502(00)00057-4

[15] The Japan Society of Mechanical Engineers (1995) JSME Combustion Handbook. Maruzen-Yushodo Co. Ltd., Tokyo, 59. (In Japanese) 


\section{Nomenclature}

$A$ : surface area of sphere

$A_{i}$ : area of the $i$-th grid

$C_{D}$ : drag coefficient

$C_{D:}$ : friction drag coefficient

$C_{D p}$ : pressure drag coefficient

$d$ diameter

$D_{\mathrm{f}}$ : friction drag force

$D_{\mathrm{p}}$ : pressure drag force

g: gravitational acceleration vector

g. gravitational acceleration

$h$ : enthalpy

$k$. thermal conductivity

$K$ : kinetic energy

$L$ : representative length

$n_{x}:$ x-direction component of unit normal vector

$p$ : pressure

$p_{i}$ : pressure of the $i$-th grid

Ra: Rayleigh number

Re: Reynolds number

$r$ radius

$r_{i x}: \mathrm{x}$-direction component of position vector of the $i$-th grid

S: projected area

T: temperature

$T_{0}$ : standard temperature

$t_{\text {end }}$ : end time of calculation

u: flow velocity vector

$\mathrm{U}$ : velocity vector of uniform mainstream

$|\mathrm{U}|$ : magnitude of velocity vector of uniform mainstream

$\mathrm{u}_{\mathrm{t} i}$ : tangential component of velocity of the $i$-th grid

$V$ : normalized maximum outflow velocity

$\alpha$ : thermal diffusivity

$\beta$ : thermal expansion coefficient

$\Delta T$ : temperature difference between particle surface and fluid

$\mu$ : viscosity

v. kinematic viscosity

$\rho$ : density at standard temperature

$\tau_{x^{x}}: \mathrm{x}$-direction component of shear stress 